\title{
XI.
}

\section{Einteilung der griechischen Philosophie.}

\author{
Von
}

Dr. Goedeckemeyer, Göttingen.

Erst dadurch, daß wir die beherrschenden Gedanken einer bestimmten Entwicklungsperiode des menschlichen Geisteslebens aus der Masse der begleitenden Erörterungen herausheben, werden wir in den Stand gesetzt, diese Periode nicht nur historisch in angemessener Weise zu bebandeln, sondern sie auch für uns selbst, für die Ausbildung unserer eigenen Welt- und Lebensanschauung fruchtbar zu machen, worin der Historiker der Philosophie stets die wichtigste Seite seiner Studien sehen wird. Das Resultat dieser Überlegungen kommt aber in der Einteilung der betreffenden Periode zum Ausdruck. Denn die Einteilung einer bestimmten Periode hat sich gerade an die jeweils leitenden und herrschenden Gedanken zu halten und sich an ihrem tatsächlich zu konstatierenden Wechsel zu orientieren, verliert aber dadurch auch die bloß subjektive Bedeutung und gestaltet sich za einem Abbilde des objektiven Geschehens aus. Wer einteilt, will nicht etwa aus Gründen der Bequemlichkeit oder der Übersichtlichkeit in dem Kontinuum des historischen Geschehens beliebige Grenzen ziehen, sondern erhebt vor allem den Anspruch, in seiner Einteilung die ihm durch die Entwicklung und den Wechsel der in dem historischen Geschehen auftretenden Richtungen des menschlichen Geisteslebens selbst aufgezwungenen Abteilungen nachzubilden. - Dabei ist kaum za bestreiten, daß er die so bestimmte Aufgabe im allgemeinen ohne große Schwierigkeiten lösen kann, weil sich im allgemeinen ziemlich leicht bestimmen läßt, wann sich die Ziele und Interessen eines historischen Kontinuums ändern und eine neue 
Richtung der Entwicklung einsetzt. Stellen sich aber doch hier und da gewisse Schwierigkeiten ein, so wird man ihrer durch das Prinzip Herr werden können, erst, aber auch immer, dann eine neue Periode beginnen zu lassen, wenn in dem historischen Verlauf klar erkannte und auch konsequent durchgedachte Gedanken auftreten, die, von den bisher verfolgten wesentlich verschieden, unmittelbar oder mittelbar zu einer entschiedenen und nachhaltigen, sei es formalen, sei es materialen Neugestaltung der Welt- und Lebensanschauung führen.

Geht man nun mit diesem Prinzip an die griechische Philosophie heran, um ihr eine sachgemäße Einteilung zuteil werden zu lassen, so kann nur ein Überblick über ihren Verlauf und ihre Entwicklung die Mittel zur Lösung dieser Aufgabe an die Hand geben. Der aber bietet, auf das Mindestmaß des Notwendigen beschränkt, folgendes Bild.

Aus der mythologischen Kosmologie, welche mit Hilfe der dichterischen Phantasie - die Frage nach dem Urzustande und der Entstehung der Welt aus diesem. Urzustande zu beantworten suchte, entstand die Philosophie dadurch, daB an die Stelle der dichterischen Phantasie die vernünftige, ihrer Gründe sich bewußte und auf systematische Vollständigkeit ausgehende Reflexion trat, und das kosmologische Problem in die Frage nach dem wahrhaft Seienden überging. Seit Thales, in dem wir mit Aristoteles den ersten Philosophen anzuerkennen haben, bedient man sich beim Aufstellen einer Weltanschauung nicht mehr der Phantasie, sondern der Vernunft, und fragt auch nicht mehr darnach, wiè es einst war, und wie der gegenwärtige Zustand der Welt daraus geworden ist, sondern gibt dieser Frage die philosophische Wendung nach dem, was der Ur- und Grundstoff, was das eigentliche Wesen der Welt und aller Dinge in ihr ist. Aber diese Frage nimmt das philosophische Interesse dieser ersten Denker nicht nur in höchstem Maße, sondern auch in allererster Linie, ja im allgemeinen sogar ganz allein in Anspruch. Was sie an anderen Problemen nicht sozusagen von selbst mit sich bringt, wie die auch erst relativ spät in Angriff genommene Frage nach der Zuverlässigkeit der Sinne, wird einfach nicht zum Gegenstande philosophischer Erörte- 
rung gemacht. So erklärt. sich die zunächst höchst auffallende Erscheinung, daß bei diesen Männern ethische Betrachtungen meist in derselben aphoristischen Form auftreten, wie sie uns in den Weisheitssprüchen der gleichzeitigen Gnomiker begegnen, oder gar, wie bei den Pythagoreern und Empedokles, eine religiös gefärbte Ethik zum Vorschein kommt, die mit den philosophischen Erwägungen nicht das Geringste zu tun hat, sondern ganz anderen Gedankenkreisen entstammt. So erklärt sich aber auch der naive Mut, mit dem sie ohne den geringsten Gedanken an eine sorgfältige Untersuchung der menschlichen Erkenntnisfähigkeit, ja ohne sich auch nur einer Methode ihrer philosophischen Erörterungen zu versichern, sogleich an die in ihrer Möglichkeit in keiner Weise angezweifelte Lösung des aufgestellten Problems herangehen.

In die naiv dogmatische Tätigkeit der Naturphilosophen griff nun mit rauher Hand die Sophistik ein, und zwar, indes wohl nur aus rein äußeren Gründen, weniger der negative Dogmatismus des Gorgias als der Relativismus des Protagoras und derer, die sich an ihn anschlossen. Sie erklärten auf der einen Seite und im Gegensatze zu dem Anspruch der Naturphilosophen, die Wahrheit gefunden zu haben, daß von einer für alle gültigen Wahrheit überhaupt keine Rede sein könne, sondern daß für jeden das sei, was ihm zu sein scheine, und verbanden hiermit die Ablehnung jeder naturphilosophischen Erörterung in dem bisher damit verknüpften Sinne; zugleich aber und obendrein veranlaßt durch die Zustände des öffentlichen Lebens stellten sie sich auf der andern Seite die ganz neue Aufgabe, vor allem durch ethische, rhetorische und dialektische, vielfach aber auch durch jede mögliche andere Belehrung ihre Vlitbürger aufzuklären und zu erfahrenen, für das private und öffentliche Leben brauchbaren Männern zu machen, was natürlich auch eine wissenschaftliche Betätigung auf diesen Gebieten zur Folge hatte und voraussetzte.

Diese von den Sophisten vertretene Richtung wurde vun in vertiếfter Form von Sokrates aufgenommen. Er ist eins mit ihnen in der Ablehnung der Naturphilosophie, weil er nicht nur meint, daß die Beschäftigung mit ihr für den Menschen völlig aussichtsund nutzlos sei, sondern auch entschieden dafür eintritt, von einer 
Beschïftigung mit solch fernliegenden Problemen erst einmal über das ins Reine zu kommen, was dem Menschen näher liege. Und eben deshalb tritt er auch darin auf ihre Seite, daß er wie sie sein ganzes Interesse dem Leben zuwendet. Aber er weicht doch in verschiedenen Punkten in bedeutungsvoller Weise von ihnen ab. Nicht nur beschränkt er .sich im Gegensatz zu der sophistischen Aufklärung ganz auf die Betrachtung des Sittlichen und dessen, was damit in Verbindung steht - bezeichnet er es doch als seine Lebensaufgabe, sich und andere besser zu machen! sondern, was wichtiger ist, er fühlt sich nicht als Wissender, sondern als Suchender. Und da er nun die Überzeugung hegt, daß nur derjenige richtig handeln kann, der ein richtiges Wissen von dem zu Tuenden besitzt, so ergab sich für ihn aus dieser Stimmung heraus und gegenüber dem Relativismus der Sophisten die doppelte Frage, was eigentlich ein solches Wissen bedeute, und wie es $\mathrm{zu}$ gewinnen sei. Die Antworten aber, die er darauf gab, sind bekannt; Wissen heißt: das Wesen oder, was auf dasselbe hinauskommt, den Begriff des zu Wissenden kennen, und gewonnen wird ein solches Wissen durch eine besondere Methode, durch die von Sokrates freilich nicht theoretisch ausgebildete, sondern nur faktisch befolgte dialektische Induktion.

Will man aber von hier aus den Fortschritt der griechischen Philosophie verstehen, so muß man seinen Blick über Sokrates hinaus zurücklenken. Durch die Sophisten war das Interesse des. denkenden Geistes von der Betrachtung der naturphilosophischen Objekte abgezogen und dem Leben zugewendet, durch Sokrates' Neuerungen war ihm ein wohldefiniertes Ziel und eine Methode zur Erreichung dieses Zieles gewiesen. Beides aber ist für die Entwicklung der griechischen Philosophie von gleicher Bedeutung gewesen. I)as erste extensiv, das zweite intensiv. Jenes bewirkt, daß man nunmehr zum ersten Male auch die Ethik philosophisch zu behandeln beginnt, dieses, daß man unter Zurückweisung des sophistischen Relativismus, der sich nach und nach auch des ethischen Gébietes bemächtigte, festhaltend an der ungeprüften Überzeugung von der Möglichkeit eines Wissens, dieses nunmehr auch seinem Begriffe nach festgelegte Wissen auf methodische Weise zu eireichen sucht. 
Dabei hat aber das spezifisch sokratische Element mehr. auf Plato und Aristoteles, das Sokrates mit den Sophisten gemeinsame dagegen mehr auf die zugleich auch wesentlich von den Sophisten selbst beeinflußten sogenannten kleineren sokratischen Schulen eingewirkt.

Fußend auf der Bestimmung des Wissens als des Erfassens des Begriffs eines Wissensobjektes kam Plato unter Ergänzung der von ihm wenigstens gelegentlich auch theoretisch behandelten sokratischen Induktion einmal durch die Methode der Division der. Begriffe und zweitens durch die einer Intuition vergleichbaren Lehre von der Wiedererinnerung und unter gleichzeitiger Benutzung parmenideischer und herakliteischer Gedanken, der Bezeichnung allein des Seienden als Gegenstands des Wissens nämlich und der Annahme des beständigen Flusses des sinnlich Wahrnehmbareu, zu der ebenso fesselnden wie unhaltbaren Zweiweltentheorie, die der zwischen Sein und Nichtsein in der Mitte schwebenden, ewig werdenden Welt der Wahrnehmungen eine nur geistig zu schauende Welt des wahrhaft Seienden, der Ideen, als wie auch immer zu denkende Ursache alles natürlichen Seins und Geschehens und als Norm alles sittlichen Handelns gegenüberstellt, während Aristoteles, bei dem die induktive Methode seines Vorgänger durch die wegen ihrer Abhängigkeit von intuitiv erkannten Prinzipien seines Erachtens ein absolut sicheres Wissen verbürgende und jetzt auch von einer besonderen Disziplin aufs eingehendste besprochene deduktive Methode, genauer noch die Nethode des apodiktischen Syllogismus ersetzt wird, trotz seines Widerspruchs gegen die Ideenlehre doch ein, wenn auch weit kühlerer und universalerer, Fortbildner des platonischen Standpunktes, das letzte, allein wahrhaft seiende Prinzip der natürlichen Tielt in der durch ihr bloßes Dasein, also als Zweckursache, wirkenden Gottheit findet und in dem Göttlichen in uns, d. h. dem menschlichen Geiste, in dem das dem Menschen im Gegensatz zu allem übrigen Lebenden eigentümliche Wesen zum Ausdruck kommt, die Norm für alle menschliche Tätigkeit zu besitzen überzeugt ist.

Die kleineren sokratischen Schulen dagegen sind dadurch charakterisiert, daß sie, ohne, wie die an eleatische Gedanken er- 
innernde metaphysische These Euklids und der wohl in erster Linie aus polemischem Motiven entstandene Nominalismus des Antisthenes zeigen, die Erörterung über das wahrhaft Seiende völlig beiseite $z u$ lassen, den eigentlichen Gegenstand der Philosophie doch in den ethischen Problemen erkennen, aus denen sich an Stelle der sokratischen Frage nach dem richtigen Handeln bei ihnen immer mehr das durch die Antwort des Sokrates freilich nahe genug gelegte und auch bei Plato und Aristoteles in der Ethik an erste Stelle gerückte Problem der Glückseligkeit als das die ganze nicht nur ethische, sondern überhaupt philosophische Erörterung Beherrschende und Bestimmende heraushebt. Darin besteht nun aber die für den weiteren Verlauf der griechïschen Philosophie wichtigste Tat. Hatte man bisher, und auch Plato und Aristoteles bilden in dieser Hinsicht keine Ausnahme, in der Frage nach dem wahrhaft Seienden das bedeutsamste Problem der Philosophie gesehen, so tritt hier die Frage nach der Glückseligkeit an die erste Stelle. Nicht mehr die Erkenntnis des wahrhaft Seienden, sondern die Frage, wie man glücklich werden kann, nimmt jetzt das Hauptinteresse des philosophischen Denkens in Anspruch.

$\mathrm{Zu}$ entschiedener Geltung kommt diese Geistesrichtung jedoch erst in der nacharistotelischen Philosophie. Und zwar ist der erste, der in dieser Zeit ganz und gar auf ihrem Boden steht, Pyrrho, der Skeptiker. Überzeugt, daß alles darauf ankommt, glücklich zu leben, sucht er den Weg ausfindig zu machen, der uns zu einem solchen glücklichen Leben zu führen vermag, und glaubt, anfangs wenigstens, nicht ohne ein Wissen um das wahrhaft Seiende auskommen zu können. Ehe er sich aber durch diese bisher maßgebende Ansicht zu der Inangriffnahme der metaphysischen Probleme bestimmen läßt, wendet er sich - und darin besteht die überragende Bedeutung dieses Mannes - erst einmal der Frage zu, ob unsere Erkenntniskräfte die ihnen zugemutete Aufgabe überhaupt zu leisten imstande sind und findet, daß davon gar keine Rede sein kann, daß weder die Sinne noch der Verstand noch beide zusammen als Kriterien der Wahrheit angesehen werden dürfen. Iamit war nun aber ein völlig neuer philosophischer Standpunkt gewonnen, ein Standpunkt, der auf der einen Seite die Beschäftigung mit allen 
Fragen, die das wahrhaft Seiende angehen, auf gute Gründe gestützt ablehnte, und auf. der andern Seite das ganze praktische Handeln des Menschen auf die Erscheinungen stellte. Und in ihm glaubte nun Pyrrho gleichzeitig das sicherste Mittel zur Glückseligkeit zu besitzen.

Darin wollten ihm nun freilich die beiden anderen. Schulen dieser Zeit - denn von dem allein naturphilosophisch interessierten Strato können wir füglich absehen - nicht recht geben, sondern hielten an der Notwendigkeit des Wissens für die Erreichung der Glückseligkeit, die auch für sie das wichtigste Problem war, fest. Hatte aber Pyrrho die Möglichkeit des Wissens entschieden geleugnet, so konnten sie nicht umhin, auf diese Frage ebenfalls einzugehen und das Kriterium der Wahrheit nachzuweisen, das sie ihrem Wissen zugrunde legen wollten. Und so sehen wir sowohl die Epikureer als auch die Stoiker eifrig mit diesem Problem beschäftigt und sich nach ihrer affirmativen Beantwortung auch wieder der Frage einer zu dem als möglich erwiesenen Wissen führenden Methode zuwenden, die jene in einer empirischen Induktion bezw. einem darauf gegründeten Analogieverfahren finden, während sich die Stoa das in gewisser Weise umgestaltete deduktive Schlußverfahren der peripatetischen Schule zu eigen macht. Und von hier aus baut dann jede der beiden Schulen, wenn auch unter weitgehendem Anschlu $ß$ an frühere naturphilosophische und ethische Ausführungen, eine vollständige Welt- und Lebensanschanung auf, die bei Epikur den Charakter eines naturphilosophisch allerdings ziemlich inkonsequenten mechanistischen JIaterialismus trägt, während sie bei den Stoikern die Form eines großartig durchdachten materialistischen Pantheismus besitzt. Aber auch dadurch unterscheiden sich die Systeme beider Schulen voneinander, daß das stoische System einen weitaus dogmatischeren Charakter trägt, weil es alle Probleme einer endgültigen Lösung entgegenzuführen strebt, während sich die aufklärerische Tendenz des Epikureismus in naturphilosophischen Fragen außerordentlich oft mit dem Nachweis der Jöglichkeit einer natürlichen Erklärung der Erscheinungen begnügt, die endgültige Lösung des Problems aber als über die Fähigkeit des Menschen hinausliegend beiseite läßt. 
Gegen diese Positionen, vor allem gegen den weitergehenden Dogmatismus der Stoa erhob nun aber wieder die Skepsis ihr Haupt, die inzwischen in der Akademie einen festen Sitz gefunden hatte. Sie betont - es ist besonders an Carneades und dessen Schule zu denken - , daß es ein Kriterium der Wahrheit nicht gebe, und fügt hinzu, daß auch die kataleptische Vorstellung der Stoiker nicht als ein solches angesehen werden dürfe, so daß von einem Wissen nach wie vor keine Rede sein.könne. Da sie aber seit Arcesilaus auch diese Grundthese selbst in den Zweifel einbezogen hatte, so wollte sie die Möglichkeit des Wissens nicht absolut verneinen, sondern erklärte nur, daß alle bisher gemachten Versuche einer Lösung der das wahrhaft Seiende betreffenden Probleme mißlungen seien, die Wahrheit daher noch weiter gesucht werden müsse. Um aber inzwischen doch wenigstens einen Maßstab nicht nur für das Handeln, sondern, wie sie, darin ebenfalls über die ältere Skepsis hinausgehend, erklärte, auch für das Forschen zu besitzen, berief sie sich auf das Wahrscheinliche, das sie in bestimmten, durch ihre überzeugende Kraft und ihre Lebhaftigkeit ausgezeichneten Vorstellungen fand, ohne da $\beta$ sich freilich von den älteren Akademikern jemand bewogen gefühlt hätte, nun mit Hilfe dieses Kriteriums ein philosophisches System aufzustellen, oder auch nur so weit aus der für sie charakteristischen Epoche herauszutrèten, daß er eine Bestimmung des Wesens der Glückseligkeit, in der auch sie das letzte Ziel des Menschen sahen, zu geben suchte.

Auf diese Stellungnahme reagiert nun die an der höheren Wertung der Ethik übrigens durchaus festhaltende Stoa, in der wir zu dieser Zeit das wichtigste dogmatische System zu erblicken haben, da die Epikureer fast ganz abseits stehen, und die Peripatetiker in der Hauptsache mit historisch-literarischen oder gelehrten Spezialstudien beschäftigt sind - auf diese Stellungnahme reagiert also die Stoa in Panaetius und Posidonius durch eine gewisse Änderung in der Auffassung des Kriteriums der Wahrheit. Sie erklärt auf der einen Seite, veranlaßt durch die skeptischen Angriffe auf die kataleptische Vorstellung, daß nicht jede derartige Vorstellung als Kriterium angesehen werden könne, sondern nur diejenige welche bei gesunden Sinnen, bei guter Wahrnehmbarkeit 
des Gegenstandes, bei angemessener Beschaffenheit des Ortes und der äußeren Umstände und bei normaler Disposition des Verstandes vollzogen wurde, mit anderen Worten durchaus einwandsfrei sei, und legt auf der anderen Seite ein besonders starkes Gewicht auf die allgemeine Übereinstimmung. Eben dieser Umstand ist aber für die Fortbildung der griechischen Philosophie von vorwiegender Bedeutung geworden. Denn stellte man der in erster Linie auf die Relativität aller Wahrnehmungen begründeten Epoche der Skepsis die These von der Übereinstimmung aller Philosophen in den wichtigsten Fragen gegenüber, so mußte der Versuch, diese These als berechtigt nachzuweisen, angesichts der faktischen Differenzen zwischen den einzelnen Schulen mit Notwendigkeit zu einer Kompromißphilosophie führen, d. h. zu einer Philosophie, die das bei den verschiedenen Schulen Übereinstimmende im Gegensatz zu dem sie Trennenden hervorhob, und außerdem dieses letztere so gering wie nur irgend möglich erscheinen zu lassen suchte. Und in dieser Richtung scheinen nun in der Tat bereits die mittleren Stoiker tätig gewesen zu sein, für die in sachlicher Hinsicht die an Plato anknüpfende Entgegensetzung von Geist und Leib charakteristisch ist. Ganz sicher ist es aber für den unter ihrem Einfluß von der skeptischen Akademie zum Dogmatismus abgefallenen Antiochus, der in der Akademie und dem Peripatos unter verschiedenen Namen nur eine einzige Schule und in der Stoa höchstens eine Verbesserung der alten Akademie, nicht aber ein durchaus neues System sehen wollte. Cnd von ihnen aus hat dann diese Kompromißphilosophie auch in die akademische Skepsis Eingang gefunden, indem Cicero unter des Antiochus Einfluß stehend mit der schon von Carneades als möglich anerkannten Systembildung auf akademisch-skeptischem Boden Ernst machte. Und ähnlich scheinen dann auch einige weitere, aber unbedeutendere Anhänger dieser Schule vorgegangen zu sein, während die jüngeren Stoiker unter immer schärferer Anspannung des schon bei ihren näheren Vorgängern vorhandenen Gegensatzes zwischen Leib und Seele mehr und mehr die Lebensansicht hinter die Lebensführung zurückstellten und die Stoa dadurch, daß sie zum Wiederaufleben des Zynismus beitrugen, schließlich wieder zu ihrem Ursprung zurückkommen ließen. 
Aber das von den letzten Anhängern der neuen Akademie beliebte Hinausgehen über den zurückhaltenden Standpunkt, der bisher in dieser Schule herrschend gewesen war, hatte nun wieder einen neuen Angriff von seiten der radikaleren Skepsis zur Folge. Aenesidem wollte in der Ǔberzeugung, daß die letzte Entwicklung der Akademie mit den von ihr bisher vertretenen Thesen in vollem Widerspruch stehe, ja, daß sie im Grunde schon von Anfang an gar nicht die echte Skepsis besessen habe, zu einer Erneuerung dieser Denkrichtung schreiten unter Anschluß an Pyrrho. Und wenn seine Skepsis auch der akademischen viel näher steht als der noch halb dogmatischen Lehre Pyrrhos, so ist sie doch auch von jener verschieden sowohl durch die auf den beständigen Wechsel der Wahrnehmungen und. Urteile gestützte Begründung ihrer Grundthese, wie auch durch die weit prinzipiellere Gestaltung des Angriffs auf die Dogmatiker, der sich nicht mehr gegen die einzelnen Thesen der dogmatischen Schulen richtet, sondern gegen die Grundannahmen des Dogmatismus selbst.

Diese letzte Erneuerung der Skepsis hatte aber einen doppelten Erfolg. Sie führte auf der einen Seite in Verbindung mit orientalischen Denkmotiven im Neuplatonismus zu einer religiös gestimmten Offenbarungsphilosophie, die gleichgültig gegen alle dialektischen und im engeren Sinne naturphilosophischen Probleme nur die das Leben des Menschen unmittelbar oder mittelbar angehenden Fragen ins Auge faßt, wie die Frage nach dem Verhältnis zwischen Gott und Welt, zwischen. Geist und Leib, und als letztes Ziel des Menschen die auf genau beschriebenem Wege schon auf Erden vorübergehend zu erreichende mystische Vereinigung des Menschen mit der weit über alle Bestimmtheit hinausgerückten Gottheit betrachtèt; auf der anderen Seite aber kommt sie in Verbindung mit der Schule der empirischen Ärzte in Menodot und Sextuis Empiricus zu einem völlig metaphysikfreien Positivismus, der, selbst eine Art des Skeptizismus, sich von der pyrrhonischèn Skepsis nur dadurch unterscheidet, dảß er die Zurückhaltung in den aus rein theoretischem Interesse behandelten metaphysischen Fragen durch das Aufstellen von $W$ issenschaften ergänzt, die sich im Dienste des Lebens auf die Konstatierung der Erschei- 
nungen und der zwischen ihnen bestehenden zeitlichen Verhältnisse beschränken. -

Suchen wir nun auf Grund dieses kurzen Überblicks über die Entwicklung der griechischen Philosophie zu einer angemessenen Einteilung derselben zu gelangen, so ist zunächst so viel klar, daß wir den wichtigsten Schnitt an der Stelle ausführen müssen, an der das Objekt des philosophischen Denkens ein völlig anderes wird, an der sich die ganze Richtung des 'philosophischen Interesses von Grund aus ändert. Das aber ist der Fall in der nacharistotelischen Philosophie. In ihr wendet sich das philosophische Denken nicht mehr wie bisher in erster Linie der Erkenntnis des wahrhaft Seienden zu, sondern macht die Frage nach dem Erreichen der Glückseligkeit zu seinem Hauptproblem, und behandelt alle übrigen Fragen nur insoweit, als sie unmittelbar oder mittelbar mit dieser wichtigen Frage in Zusammenhang stehen. Und so werden wir den Tod des Aristoteles als den Scheidepunkt zwischen den beiden Hauptperioden des griechischen Philosophie anzusehen haben.

Bei der Untereinteilung dieser Hauptabschnitte werden wir uns aber nicht mehr an die materiale, sondern an die formale Seite der Philosophie halten müssen. Dann tritt uns innerhalb des ersten Hauptabschnittes als wichtigste Differenz der Umstand entgegen, daß man sich an die Behandlung des zu lösenden Problems zunächst völlig unmethodisch heranmacht, während man sich seit Sokrates' Auftreten einer zuvor festgelegten Methode bedient. Und so werden wir jedenfalls zwischen dem naiven und dem methodischen Dogmatismus zu unterscheiden haben.

Zweifelhaft kann es nur sein, zu welcher Periode wir die Sophisten rechnen sollen. Da sie jedoch in ihrer ganzen philosophischen Stellung jedenfalls eine größere-Verwandtschaft mit Sokrates besitzen als mit dem Naturphilosophen, da sich ferner insbesondere Protagoras durch seine wissenstheoretischen Erörterungen viel mehr als Vorläufer des Sokrates als durch seine naturphilosophischen Thesen als Abschluß der vorhergehenden Naturphilosophie erweist, so werden wir besser tun, sie zur zweiten Periode des ersten Hauptabschnittes zu zählen. 
Innerhalb des zweiten Hauptteiles aber sind die Perioden ohne weiteres durch den Streit $z$ wischen dem radikalen und die strengste Zurückhaltung fordernden Skeptizismus auf der einen Seite, dem Dogmatismus und dem wie dieser positiven Entscheidungen geneigten Skeptizismus auf der anderen Seite gegeben. Denn dem jede Möglichkeit der Erkenntnis leugnenden Skeptizismus Pyrrhos tritt der Dogmatismus mit dem Hinweis auf das Kriterium der Wahrheit entgegen; auf den dieses Kriterium zurückweisenden, aber seine eigene These selbst einschließenden und daher auch die Möglichkeit der Erkenntnis zugestehenden carneadeischen Skeptizismus antwortet der Dogmatismus mit der Zurückziehung auf die auf die allgemeine Übereinstimmung begründete Kompromißphilosophie, die unter der Flagge einer nur wahrscheinlichen Geltung auch in die Skepsis eindringt; und die durch eine leichtverständliche Begründung und durch scharfsinnige Bekämpfung der dogmatischen Prinzipien ausgezeichnete Skepsis Aenesidems endlich führt den Dogmatismus zur Ergänzung seines Wissens durch die Offenbarung, und die positiv gerichteten Skeptiker zum Positivismus. Und so werden wir schließlich als das Resultat der angestellten Überlegung die griechische Philosophie folgendermaßen einzuteilen haben :

I. Die ontologische Periode:

1. Der naive Ontologismus;

2. Die Sophisten und der methodische Ontologismus.

II. Die eudaemonologische Periode:

1. Die pyrrhonische Skepsis und Epikur und die Stoa;

2. Die carneadeische Skepsis und die Kompromißphilophie;

.3. Die aenesidemische Skepsis und die Offenbarungsphilosophie und der Positivismus. 